\title{
RESEARCH HIGHLIGHT To learn something new, do something new
}

\author{
Adam M. P. Miller ${ }^{1}$ and Paul W. Frankland (D) $1,2,3,4,5$ \\ Cell Research (2021) 31:611-612; https://doi.org/10.1038/s41422-021-00508-7
}

\begin{abstract}
How do we free ourselves from outdated routines when conditions change? Park et al. show that a novel experience can weaken old connections within the brain's memory system, thereby freeing up resources to learn something new.

Routines are common solutions for exploiting predictable environments, but they can become a burden if the environment changes. For example, many people settle into a commuting routine whereby they leave at a specific time and follow a specific route. However, if a new construction project disrupts the route, the routine needs to be abandoned in favor of something new (Fig. 1). Failing to detect the change or persisting in the old routine makes it difficult to learn a new one.
\end{abstract}

Fortunately, the brain's memory system is sensitive to these kinds of changes. Rodents form new representations in the hippocampus, prefrontal cortex and elsewhere when entering a new environment. $^{1-3}$ They can also generate new representations when rules change ${ }^{4,5}$ or update existing representations with new information. ${ }^{6,7}$ Detecting these changes requires identifying mismatches-known as prediction errors-between what the mouse expects, such as a familiar experience, and what the mouse encounters, such as a novel one. ${ }^{8}$ Prediction errors play a key role in learning by identifying what is important to learn. However, they can also signal that the current routine is outdated and needs replacing.

In a new paper published in Nature, Park et al. ${ }^{9}$ investigate the role that novelty plays in enabling mice to abandon an outdated routine when conditions change. The authors first allowed mice to settle into a routine whereby they repeatedly retrieved rewards from one arm of a T-maze. The rules were then changed so that the location of the reward alternated between trials. The authors manipulated novelty by briefly placing the mice into another room separate from the training environment that was either completely new (novel experience) or that had been visited prior (familiar experience). Mice given a novel experience before training showed evidence of learning the new rule, whereas mice given a familiar experience persisted in their outdated routine.

Using in vivo electrophysiology, the authors directly observed the representational shift as mice switched strategies. They focused on regions known to support memory retrieval and new learning, including the ventral hippocampus (vHPC) and the medial prefrontal cortex (mPFC). Early in training, a classifier applied to activity in the vHPC decoded trials based on whether the mouse was about to enter the arm of the T-maze associated with its now-outdated routine. However, after exposure to a novel environment, activity in the VHPC slowly changed until it encoded reward locations according to the new rule.

The authors then asked what changes were brought about by the novel experience that might support the acquisition of the new strategy. After visiting a novel environment-but not a familiar one-mice showed increased VHPC theta power accompanied by a reorganization of neural activity toward the troughs of the theta cycle. This suggests that the vHPC may weaken existing synaptic connections, ${ }^{10}$ and, consistent with this, the authors also observed decreased mPFC responses to vHPC neurons and reduced phase locking of mPFC activity to VHPC theta.

Interestingly, whereas novelty decreased vHPC-mPFC coordination, learning the new strategy reinstated it: after learning, vHPCmPFC coordination returned to the levels observed before the novel experience. This suggests that novelty cleared the decks, so to speak, to provide space for new learning. One possibility is that novelty de-potentiated synapses to make room for the new plasticity required to learn the new strategy. To test this, the authors manually potentiated VHPC-mPFC connections and then exposed the mice to a novel environment. As predicted, the novel experience de-potentiated the synapses that were just manually potentiated. Furthermore, the authors found that manually potentiating the synapses after a novel experience-canceling out the de-potentiating effects of novelty-prevented new learning.

Together, this suggests that novelty may act via vHPC neurons to weaken vHPC-mPFC connectivity. Because dopaminergic projections from the ventral tegmental area signal novelty to the $\mathrm{HPC}^{11}$ one possibility is that this effect is mediated by vHPC neurons expressing dopamine receptors. Consistent with this, the authors found that activating D1 receptors mimicked the effects of exposure to a novel environment, whereas blocking D1 receptors prevented the same effects. The authors confirmed the importance of novelty-responsive VHPC neurons in this process by tagging and inhibiting them for $10 \mathrm{~min}$ immediately after exposure to novelty. This brief inhibition prevented the mice from acquiring the new strategy.

This impressive work from Park et al. illustrates how novelty prepares mice to change their behavior by resetting interactions between the VHPC and the MPFC. But why should novelty control the reset switch? It may have to do with the classic explorationexploitation dilemma, whereby animals must determine when to stop spending resources learning about their environment and start using what they already know to exploit it. To solve this problem, animals may monitor their ability to predict ongoing experience; they are ready to exploit an environment once they can predict the events occurring within it. On the other hand, a novel or otherwise unpredicted event might shift the system back toward exploration.

A new environment is the quintessential novel experience in this regard because new environments often have new rules. However, the effect of novelty is probably weaker for prediction errors occurring in the same environment (or else we might

${ }^{1}$ Program in Neurosciences and Mental Health, Hospital for Sick Children, Toronto, ON M5G 1X8, Canada; ${ }^{2}$ Department of Psychology, University of Toronto, Toronto, ON

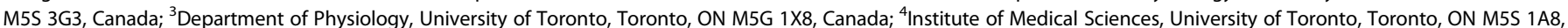
Canada and ${ }^{5}$ Child and Brain Development Program, Canadian Institute for Advanced Research, Toronto, ON M5G 1M1, Canada

Correspondence: Adam M. P. Miller (adam.miller@sickkids.ca) or Paul W. Frankland (paul.frankland@sickkids.ca)

Published online: 28 April 2021 


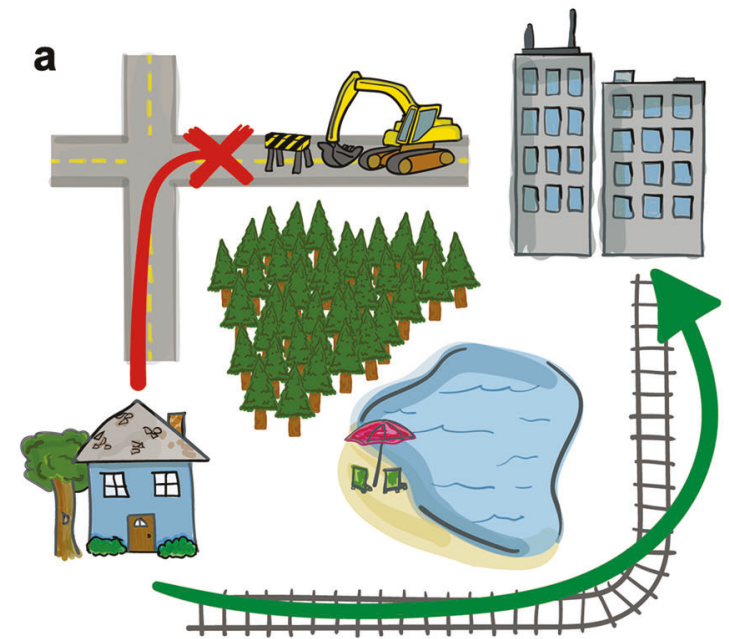

b

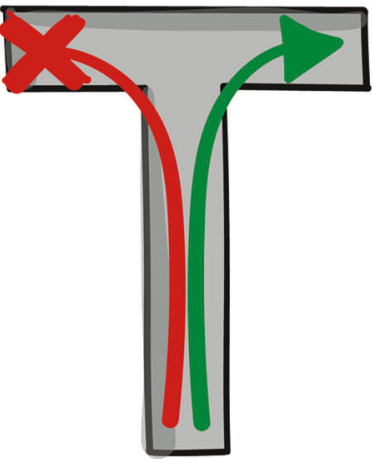

Fig. 1 A change in environmental conditions requires updating routines. a If construction interferes with your commuting routine, then you must find an alternative. $\mathbf{b}$ After learning to repeatedly retrieve rewards from one arm on a T-maze, the rules changed, and mice needed to learn to alternate.

abandon our commute every time we encounter a new traffic sign). This may explain why the mice in the familiar condition persisted with their outdated routine even after the rules changed: the prediction errors arising from missing rewards were insufficient to produce the full novelty response. Future work quantifying and controlling the degree of novelty experienced by the subject-either behaviorally in terms of the mismatch between existing knowledge and new information, or physiologically in terms of the magnitude of novelty-related inputs-could explore the boundary conditions determining whether a subject persists in their current strategy, modifies it with new information, or abandons it entirely.

\section{REFERENCES}

1. Muller, R. U. \& Kubie, J. L. J. Neurosci. 7, 1951-1968 (1987).
2. Hyman, J. M., Ma, L., Balaguer-Ballester, E., Durstewitz, D. \& Seamans, J. K. Proc. Natl. Acad. Sci. USA 109, 5086-5091 (2012).

3. Miller, A. M. P., Serrichio, A. C. \& Smith, D. M. Cereb. Cortex. https://doi.org/ 10.1093/cercor/bhaa386 (2020).

4. Durstewitz, D., Vittoz, N. M., Floresco, S. B. \& Seamans, J. K. Neuron 66, 438-448 (2010).

5. Bulkin, D. A., Law, L. M. \& Smith, D. M. Hippocampus 26, 958-971 (2016).

6. Lee, J. L. C., Nader, K. \& Schiller, D. Trends Cogn. Sci. 21, 531-545 (2017).

7. Gava, G. P. et al. Nat. Neurosci. 24, 326-330 (2021).

8. Rescorla, R. A. \& Wagner, A. A theory of Pavlovian conditioning: variations in the effectiveness of reinforcement and nonreinforcement. in Classical Conditioning II: Current Research and Theory (eds Black, A. H. \& Prokasy, W. F.) (Appleton-CenturyCrofts, 1972).

9. Park, A. J. et al. Nature 591, 615-619 (2021).

10. Hyman, J. M., Wyble, B. P., Goyal, V., Rossi, C. A. \& Hasselmo, M. E. J. Neurosci. 23, 11725-11731 (2003).

11. Lisman, J. E. \& Grace, A. A. Neuron 46, 703-713 (2005). 